\title{
Therapeutic effects of ciprofloxacin/bushenhuazhuo combination on chronic prostatitis
}

\author{
Xiao-Wei Qu*, Zhong-Jie Shan, Nan Zhang and Guo Liang \\ Urology Surgery Dept., People's Hospital of Zhengzhou, Zhengzhou, 450003, PR China \\ ${ }^{*}$ For correspondence: Email: xiaoweiqxw@163.com
}

Received: 27 April 2016

Revised accepted: 20 August 2016

\begin{abstract}
Purpose: To evaluate the clinical effect of bushenhuazhuo (a Chinese traditional medicine) in combination with ciprofloxacin (an orthodox medicine) in chronic prostatitis (CP) therapy.

Methods: A total of 160 patients who suffered from CP and received treatment in the People's Hospital of Zhengzhou between April 2012 and June 2014 were selected and divided randomly into treatment and control groups, with 80 patients in each group. Control group was given $0.25 \mathrm{~g}$ ciprofloxacin hydrochloride tablets twice a day for 4 weeks. In addition to ciprofloxacin administration, patients in the treatment group also received a dose of bushenhuazhuo preparation twice daily for 4 weeks. Clinical outcomes, quality of life as well as lecithin body and white blood cell (WBC) count in expressed prostatic secretions (EPS-WBC) were evaluated.

Results: Cure rates in the treatment and control groups were 90 and $72.50 \%$, respectively; this difference was significant $(p<0.05)$. Scores for National Institutes of Health Chronic Prostatitis Symptom Index (NIH-CPSI), WBC, and lecithin bodies in the treatment group (8.20 \pm 2.20 points, $4.50 \pm$ 1.20 points, and $28.10 \pm 2.10$ points, respectively) were higher $(p<0.05)$ than for the control group $(12.20 \pm 2.20,6.30 \pm 2.20$, and $23.30 \pm 2.90$ points, respectively). The levels of interferon (IFN) $-y$ and tumour necrosis factor (TNF)- $\alpha$ in the treatment group $(26.20 \pm 3.30$ and $33.80 \pm 5.40 \mathrm{mg} / \mathrm{L}$, respectively) were lower than those in the control group $(37.70 \pm 3.90$ and $48.40 \pm 3.70 \mathrm{mg} / \mathrm{L}$, respectively), whereas the level of interleukin (IL)-10 in the treatment group (292.60 $\pm 23.70 \mathrm{mg} / \mathrm{L})$ was higher $(p<0.05)$ than that in control group $(235.80 \pm 25.90 \mathrm{mg} / \mathrm{L})$.

Conclusion: Ciprofloxacin combined with the Chinese traditional medicine, bushenhuazhuo preparation, demonstrates a marked therapeutic effect in $C P$. Its mechanism of action may be related to decreased levels of IFN- $y$ and TNF- $\alpha$ and increased IL-10.
\end{abstract}

Keywords: Bushenhuazhuo, Interferon - v, Ciprofloxacin, Chronic prostatitis symptom index

Tropical Journal of Pharmaceutical Research is indexed by Science Citation Index (SciSearch), Scopus, International Pharmaceutical Abstract, Chemical Abstracts, Embase, Index Copernicus, EBSCO, African Index Medicus, JournalSeek, Journal Citation Reports/Science Edition, Directory of Open Access Journals (DOAJ), African Journal Online, Bioline International, Open-J-Gate and Pharmacy Abstracts

\section{INTRODUCTION}

Chronic prostatitis (CP), one of the most commonly seen diseases in the male urogenital system, accounts for one-third of men's diseases; it usually occurs in the age range of 20 - 40 years, develops slowly, and is difficult to cure. It can impact severely the quality of life of sufferers $[1,2]$. CP features a long course, common recurrence, and a complex pathological mechanism; the symptoms of paruria and perineal pain clinically can result in sexual dysfunction and infertility if the treatment is delayed $[3,4]$. The effects of currently available Western medicines have not been satisfactory.

Modern medicine considers that $\mathrm{CP}$ can be divided into chronic bacterial prostatitis, non- 
bacterial prostatitis, and prostatodynia according to the Meares - Stamey classification [5]. In 1995, the United States National Institutes of Health (NIH) proposed dividing prostatitis into category I, category II (chronic bacterial prostatitis), category III (chronic pelvic pain syndrome or chronic abacterial prostatitis), and category IV (asymptomatic inflammatory prostatitis) [6]. Category III accounts for $90 \%$ of cases.

Traditional Chinese medicine classifies CP into the categories of Lin and Zhuo, or the categories of kidney vacuity and lumbar pain, impotence, prospermia, and retention of urine. Traditional Chinese medicine considers $\mathrm{CP}$ as induced primarily by insecurity of the essence gate, stagnation of humid heat, and liver depression. Based on the clinical experience, we used a Chinese traditional medicine, bushenhuazhuo preparation, in combination with ciprofloxacin to treat $\mathrm{CP}$ in men.

\section{EXPERIMENTAL}

\section{General data}

A total of $160 \mathrm{CP}$ patients who received treatment at the People's Hospital of Zhengzhou between April 2012 and June 2014 were selected randomly and divided into control and treatment groups, 80 in each. In the treatment group, patients aged from 29 to 64 years (mean $38.70 \pm 10.20$ years) had developed CP over the previous 2 months to 6 years (mean $1.50 \pm 0.60$ years) and in the control group, patients aged from 28 to 62 years (average $36.90 \pm 8.20$ years) had developed CP over the previous 3 months to 7 years (average $1.70 \pm 0.50$ years).

Patients who met the diagnostic criteria of CP [8], underwent no other treatment or had stopped receiving another treatment for more than 1 week, and signed an informed consent were included. Patients with a history of treatment for benign prostatic hyperplasia, prostate cancer, deformity of the urethra or prostate, or laser, radio-frequency, microwave, or ablation treatment, who had acute onset of CP and who developed severe cardiovascular and cerebrovascular diseases or liver and renal diseases were excluded. No significant difference was found in general data $(P>0.05)$; thus, the results were comparable. This study was approved by the medical ethics committee of People's Hospital of Zhengzhou (approval no. PHZ20150425QXW). It was conducted according to the tenets of the Declaration of Helsinki [9].

\section{Therapeutic regimen}

Patients in the control group were administered ciprofloxacin hydrochloride tablets orally (Tianfang Pharmacy Inc., Henan; branch no. H41024142), $0.25 \mathrm{~g}$ twice a day for 4 weeks. In addition to ciprofloxacin therapy, patients in the treatment group were also given bushenhuazhuo preparation. Bushenhuazhuo preparation was comprised of prepared rehmannia root $(20 \mathrm{~g})$, fructus psoraleae $(10 \mathrm{~g})$, corydalis yanhusuo (10 $\mathrm{g})$, radix cyathulae $(15 \mathrm{~g})$, semen plantaginis (15 g), Dioscorea tokoro Makino (10 g), herba patriniae (15 g), golden cypress (10 g), rhizoma acori graminei (10 g), Poria cocos (20 g), bighead atractylodes rhizome (15 g), salviae miltiorrhizae (15 g), and lotus plumule (10 g); a dose each was administered orally twice daily. Again, 4 weeks was regarded as one course. Patients were forbidden from taking irritable foods and were asked to take regular rest during treatments.

\section{Observation index}

The NIH Chronic Prostatitis Symptom Index (NIH-CPSI) was used. Moreover, conventional microscopy of prostate fluid was performed; specifically, the numbers of white blood cells (WBCs) and lecithin bodies were determined in five high-power fields. Levels of interferon (IFN)$\mathrm{Y}$, tumour necrosis factor (TNF)- $\alpha$, and interleukin (IL)-10 in prostate fluid were detected with enzyme linked immunosorbent assays (ELISAs) using kits (Wuhan Boster Bioengineering, Inc.). The values above were evaluated once before and after treatment.

\section{Criteria for a therapeutic effect}

The comprehensive therapy standard, based on $\mathrm{NIH}-\mathrm{CPSI}$ [10], was adopted. If the $\mathrm{NIH}-\mathrm{CPSI}$ score declined by not less than $90 \%$, prostate fluid was found to be normal in two examinations, pressure pain disappeared, and the results of bacterial cultures were negative, then $\mathrm{CP}$ was considered 'cured.' If the NIH-CPSI score decreased by not less than $60 \%$, pressure pain improved significantly, the levels of WBCs in prostate were reduced to not less than $60 \%$ in two examinations, and the result of bacterial cultures were negative, then the treatment was considered to be markedly effective.

If the $\mathrm{NIH}-\mathrm{CPSI}$ score decreased by more than $30 \%$, pressure pain was relieved, the levels of WBCs in prostate fluid was reduced by not less than $30 \%$ in two consecutive examinations, and bacteria grew, then the treatment was considered effective. If the $\mathrm{NIH}-\mathrm{CPSI}$ score was 
reduced by less than $30 \%$, pressure pain showed no improvement, the levels of WBCs in prostate fluid declined by less than $30 \%$ in two examinations, and bacteria grew, then the treatment was considered ineffective. The equation for response rate is as follows:

$d=[(a+b+c) / n] \times 100$

where $\mathrm{d}$ is the response rate, $\mathrm{a}$ is the number of 'cured' patients, b is the number of patients obtaining a significant effect, $c$ is the number of patients obtaining an effect, and $n$ is the total number of patients.

\section{Statistical analysis}

The SPSS software (ver. 19.0) was used for all analyses. The data are expressed as mean \pm standard deviation (SD). Comparison between groups was performed using t-test. Numerical data were processed using $x^{2}$ tests. Correlations were analysed using Spearman's correlation analysis. Differences were considered statistically significant if $p<0.05$.

\section{RESULTS}

\section{Comparison of therapeutic effect}

Table 1 shows that 50 patients were cured, 18 cases were significantly effective, 4 cases were effective, and 8 cases were ineffective in the treatment group, and 34 patients were cured, 10 cases were significantly effective, 14 cases were effective, and 22 cases were ineffective in the control group. The total response rate of the treatment group $(90 \%)$ was higher than that of the control group; the difference was statistically significant $(p<0.05)$.

\section{NIH-CPSI scores before and after treatment}

Table 2 shows that NIH-CPSI scores in both groups declined significantly after treatment, and the scores in the treatment group were lower than those of the control group after treatment $(p$ $<0.05)$. No significant difference was found in scores for pain, discomfort, or urinary symptom, but the score for quality of life showed a significant difference between the groups ( $p<$ 0.05).

\section{WBCs and lecithin bodies}

Table 3 shows that the numbers of WBCs in the treatment group were lower than those in the control group; the difference was significant $(p<$ 0.05). Moreover, the number of lecithin bodies in the treatment group was higher than in the control group $(p<0.05)$.

\section{IFN-y, TNF- $\alpha$ and IL-10 levels before and after treatment}

Table 4 shows that levels of IFN- $y$, TNF- $\alpha$, and IL-10 improved in both groups after treatment ( $p$ $<0.05)$. The improvement in the treatment group was more obvious than in the control group $(p<$ 0.05).

Table 1: Comparison of clinical effect between two groups ( $n)$

\begin{tabular}{lcccccc}
\hline Group & $\boldsymbol{n}$ & Cure & Very effective & Effective & Ineffective & Total cure rate (\%) \\
\hline Control & 80 & 34 & 10 & 14 & 22 & 72.50 \\
Treatment & 80 & 50 & 18 & 4 & 8 & $90.00^{*}$ \\
\hline
\end{tabular}

Note: ${ }^{*} P<0.05$, vs. control group

Table 2: NIH-CPSI scores before and after treatment (mean \pm SD)

\begin{tabular}{lccccc}
\hline Group & Time & $\begin{array}{c}\text { Pain or } \\
\text { discomfort }\end{array}$ & $\begin{array}{c}\text { Urinary } \\
\text { symptom }\end{array}$ & Quality of life & Total \\
\hline Control & Before & $13.20 \pm 2.90$ & $5.00 \pm 1.30$ & $8.80 \pm 2.20$ & $16.10 \pm 4.70$ \\
$(n=80)$ & After & $6.80 \pm 2.40 \Delta$ & $4.90 \pm 1.40 \Delta$ & $4.10 \pm 1.90^{\Delta}$ & $12.20 \pm 2.20 \Delta$ \\
Treatment & Before & $12.50 \pm 3.20$ & $4.20 \pm 1.60$ & $7.20 \pm 2.20$ & $15.20 \pm 4.70$ \\
$(n=80)$ & After & $4.60 \pm 2.30 \Delta$ & $3.10 \pm 1.40 \Delta$ & $1.80 \pm 1.00 \Delta^{\#}$ & $8.20 \pm 2.20 \Delta^{\#}$ \\
\hline Note: $\Delta P<0.05$, vs. before treatment (within group): ${ }^{\#} p<0.05$, vs. control group after treatment.
\end{tabular}

Table 3: WBCs and lecithin bodies in expressed prostatic secretions (EPS) before and after treatment (mean \pm $\mathrm{SD}, n / \mathrm{HP})$

\begin{tabular}{lcccc}
\hline Group & $\boldsymbol{n}$ & Time & WBCs & Lecithin bodies \\
\hline \multirow{2}{*}{ Control } & 80 & Before & $15.80 \pm 2.80$ & $7.30 \pm 2.20$ \\
& 80 & After & $6.30 \pm 2.20 \Delta$ & $23.30 \pm 2.90 \Delta$ \\
Treatment & 80 & Before & $16.10 \pm 3.10$ & $7.20 \pm 2.50$ \\
& 80 & After & $4.50 \pm 1.20 \Delta^{\#}$ & $28.10 \pm 2.10 \Delta^{\#}$ \\
\hline
\end{tabular}

Note: $\Delta P<0.05$, vs. before treatment (within group), ${ }^{\#} P<0.05$, vs. control group after treatment 
Table 4: IFN- $\gamma$, TNF- $\alpha$ and IL-10 levels in prostate fluid before and after treatment (mean \pm SD, mg/L)

\begin{tabular}{lccccc}
\hline Group & $\boldsymbol{n}$ & Time & IFN-y & IL-10 & TNF- $\boldsymbol{\alpha}$ \\
\hline \multirow{2}{*}{ Control } & 80 & Before & $44.50 \pm 4.70$ & $188.10 \pm 23.50$ & $71.70 \pm 6.90$ \\
& 80 & After & $37.70 \pm 3.90^{\Delta}$ & $235.80 \pm 25.90^{\Delta}$ & $48.40 \pm 3.70 \Delta$ \\
\multirow{2}{*}{ Treatment } & 80 & Before & $46.50 \pm 4.60$ & $184.50 \pm 28.40$ & $70.30 \pm 7.50$ \\
& 80 & After & $26.2 \pm 3.30 \Delta^{\#}$ & $292.6 \pm 23.70 \Delta^{\#}$ & $33.80 \pm 5.40 \Delta^{\#}$ \\
\hline
\end{tabular}

Note: $\Delta P<0.05$, vs. before treatment (within group), ${ }^{\#} p<0.05$, vs. control group after treatment

\section{DISCUSSION}

The pathogenesis of $\mathrm{CP}$ remains controversial. Most scholars consider that the occurrence of CP is associated with infection by pathogenic microorganisms, paruria, stimulation of urine, anxious emotions, neuroendocrine dyscrasia, immune reaction abnormalities, oxidative stress, and pelvic disease [11]. Unconventional sexual activities, excessive drinking, long-term intake of irritable foods, fatigue, and long-term extrusion of pelvic floor muscles are also considered to be associated with $\mathrm{CP}$. In clinical practice, antibacterial treatment is the major therapy for prostatitis; however, its curative effect is not satisfactory, which may be due to the unique anatomical structure of the prostate. Other factors, such as redundant prepuce and iatrogenic injuries, such as in catheterisations, may also induce CP. CP is associated with drug permeation inhibition and inflammatory secretions induced by the barrier function of the prostatic capsule [12].

Incorrect use of anti-bacterial drugs will weaken their curative effect and may even lead to dysbiosis and liver and kidney damage. As the effects of antibiotics alone are limited, currently, traditional Chinese medicines in combination with Western medicines are used extensively in treating CP. In the view of traditional Chinese medicine, CP belongs to categories of turbid semen, turbid urine, and stranguria and is induced by the long-term existence of wet-heat, the stagnation of qi, and blood stasis [13]. Traditional Chinese medicine is effective in the treatment of $\mathrm{CP}$. It has been shown that traditional Chinese medicines can resist inflammation, infection, and fibrosis, improving microcirculation, urodynamics, and immunity, and regulation of the $\mathrm{pH}$ value of prostatic fluid [14].

It has been shown that essence exists in the kidney and spleen; qi deficiency can result in interior dampness accumulation and turbidity; pain is induced by wetness and heat, and kidney qi damage can result in seminal emissions [15]. CP may be induced by smoking, excessive drinking, masturbation, the withdrawal method, and frequent feculent sexual intercourse. The pathogenesis of $\mathrm{CP}$ includes wet-heat, stasis, essence stasis, and kidney deficiency. Wet, heat, stasis, and qi deficiency are all involved in the pathological process of CP. Wet-heat dominates in the early stage of CP; stasis and qi deficiency dominate in the middle and later stages of CP.

Prepared rehmannia root can benefit the essence and fill the marrow. Fructus psoraleae can the warm kidney and support yang. Semen plantaginis, herba patriniae, Dioscorea tokoro Makino, golden cypress, and rhizoma acori graminei, which can clear heat, eliminate wetness, and relieve pain, can effectively relieve the clinical symptoms of prostatitis. Moreover, Poria cocos can clear dampness, tonify the spleen, calm the nerves, and quiet the heart. Bighead atractylodes rhizome can tonify the qi and spleen and eliminate wetness. The combination of the two drugs can function in tonifying the spleen and eliminating dampness [16]. Corydalis yanhusuo can promote qi to activate the blood, and salviae miltiorrhizae can promote blood circulation to remove blood stasis; they are both useful in improving blood circulation [17]. Radix cyathulae can remove blood stasis and stimulate menstrual flow. Lotus plumule, which can clear heart heat, calm liver heat, and remove spleen heat, is effective in relieving patient anxiety [18]. The preparation has functions of tonifying qi and the kidney, eliminating wetness, clearing heat, activating the blood, and dispersing blood stasis. This study showed that the treatment group had a higher total response rate than the control group; moreover, scores for quality of life and levels of WBCs and lecithin bodies in the treatment group were superior to those in the control group.

Microscopic research of $\mathrm{CP}$ has become a hotspot of research, and cell factors are key [19]. In this study, an initial exploration was made regarding the mechanism of action of $\mathrm{CP}$ treatment by assessing levels of IFN- $\gamma$, TNF- $\alpha$, and IL-10 before and after treatment. Cell factors function in regulating the immune response and mediating inflammatory reactions. IFN- $\gamma$ and TNF- $\alpha$, secreted by Th1 cells, are proinflammatory, whereas IL-10, secreted by Th2 cells, is anti-inflammatory. The balance between Th1 and Th2 can ensure the immune function of the human body and influence pathological development and transformation [20]. 
IFN- $\mathrm{Y}$ can induce the activation of macrophages; the active substances secreted can damage endothelial cells, strengthen the immune response, and promote the progression of inflammatory reactions. TNF- $\alpha$ can promote the adhesion of neutrophil granulocytes on endothelial cells and mediate the generation of adherence factor and inflammatory mediators [21]. IL-10 can inhibit $T$ cell - mediated inflammatory response. It has been found that the level of IL-10 correlates with clinical symptoms in patients [22]; the study suggested that bushenhuazhuo preparation could lower levels of IFN- $\gamma$ and TNF- $\alpha$ and increase the level of IL-10, which may be an important mechanism worthy of further study.

\section{Limitation of the study}

The number of cases in the present study was small, which may have influenced the results. Thus, studies with larger sample sizes should be carried out in the future.

\section{CONCLUSION}

Bushenhuazhuo preparation, a Chinese traditional medicine, can significantly improve local microcirculation, relieve local symptoms induced by $\mathrm{CP}$, and enhance the localised concentration of orally administered ciprofloxacin in the prostate. The drug combination did not cause any adverse reaction.

\section{DECLARATIONS}

\section{Acknowledgement}

The authors sincerely thank all who supported this work.

\section{Conflict of Interest}

No conflict of interest associated with this work.

\section{Contribution of Authors}

The authors declare that this work was done by the authors named in this article and all liabilities pertaining to claims relating to the content of this article will be borne by them.

\section{REFERENCES}

1. Xie XB, Yu SJ, Peng LK. Research progress in the pathomechanism of neurogenic inflammation in chronic prostatitis/chronic pelvic pain syndrome. Chin J Urol 2008; 29(8): 573-575.
2. Wang $Q$, Chen YD, Liu YR. Research progress of pain mechanism of chronic prostatitis. J Bethune Military Med Coll 2011; 9(5): 361-363.

3. Han CJ, Dong ZL. Research progress of male sterility mechanism in chronic prostatitis. Chin J Androl 2014; (7): $69-72$.

4. Zhang LH, Guo J, Wu ZY. Clinical study on qianliean granule for the treatment of chronic prostatitis. J Henan Univ Chin Med 2012; 27(12): 1661-1662.

5. Weidner W, Naber KG. Prostatitis syndrome: consensus of the 6th International Consultation in Paris, 2005. Aktuelle Urol 2006; 37(4): 269-271.

6. Wagenlehner FM, Naber KG. Prostatitis: the role of antibiotic treatment. World J Urol 2003; 21(2): 105-108.

7. $X u J Z$, Shen $X H, X u P H$. Clinical observation of danshen tablets in the treatment of chronic prostatitis. Chin Tradit Pat Med 2006; 28(1): 152-153.

8. Nickel JC, Nyberg LM, Hennenfent M. Research guidelines for chronic prostatitis: consensus report from the first National Institutes of Health International Prostatitis Collaborative Network. Urol 1999; 54(2): 229233.

9. Declaration of Helsinki. The 59th World Medical Conference, 2008.

10. Shi Z. Clinical effect of integrated Chinese and western medicine for chronic prostatitis. New J Tradit Chin Med 2014; 46(6): 89-91.

11. Huang $X Y$, Zhang $X K$. Experimental study of modified yinchenhao decoction on chronic abacterial prostatitis. Tianjin J Tradit Chin Med 2011; 28(5): 414-417.

12. Li XS. Clinical observation on the treatment of chronic non-bacterial prostatitis by integrative by integrative traditional Chinese medicine and western medicine. $J$ Henen Univ Chin Med 2012; 27(2): 241-242.

13. $\mathrm{Li} \mathrm{CC}, \mathrm{Hu} H X$. Clinical efficacy evaluation on the treatment of chronic non-bacterial prostatitis with integrated Chinese-western therapy. J Gen Hosp Air Force, PLA 2012; 28(2): 72-75.

14. Chen ZQ, Fu YW. Progress of chronic prostatitis. J Guangzhou Univ Tradit Chin Med 1998; 15(3): 234-237.

15. Chen TW, Yang JH. Clinical observation on 40 cases of chronic prostatitis treated with Chinese and western medicine. Jiangxi J Tradit Chin Med 1998; 29(3): 13.

16. Shen SL. Professor Zhai Yachun's experience in treating chronic prostatitis with traditional Chinese medicine. Jilin $J$ Tradit Chin Med 2002; 22(6): 8-9.

17. $L i C C, H u H X$. Clinical efficacy evaluation on the treatment of chronic non-bacterial prostatitis with integrated Chinese-western therapy. J Gen Hosp Air Force, PLA 2012; 28(2): 72-75.

18. Lu ZJ, Zhu LL. Combination of Chinese and western medicine treatment of 42 cases of chronic non-bacterial prostatitis. Henan Tradit Chin Med 2013; 33(5): 752753.

19. Xiao YP, Fan $K$, Wang B, Huang M. Clinical observation of oxalis on IL-1 $\beta$, TNF- $\alpha$ levels of type III prostatitis. Guide Chin Med 2013; 11(28): 195-196.

Trop J Pharm Res, September 2016; 15(9): 2021 
20. $L u$ YH, Zhang ZG, Li JC. Clinical significance of determination of changes of serum $I L-1 \beta, I L-1 \beta$ and TNF- $\alpha$ levels after treatment in patients with chronic prostatitis. J Radioimmanol 2009; 22(6): 593-594.

21. Wang HZ, Liu CD, Wei C. The role of IFN-y, TNF- $\alpha$ and IL-10 in the rat's model for chronic abacterial prostatitis. J Chongqing Med Univ 2009; 34(9): 1187-1190.
22. Miller LJ, Fischer KA, Goralnick SJ, Litt M, Burleson JA, Albertsen $P$, Kreutzer $D L$. Interleukin-10 levels in seminal plasma: implications for chronic prostatitischronic pelvic pain syndrome. J Urol 2002; 167(2 Pt 1): 753-756. 